Article

\title{
Acute Cytauxzoon felis Cases in Domestic Cats from Eastern Kansas, a Retrospective Case-Control Study (2006-2019)
}

\author{
Yvonne M. Wikander ${ }^{1} \mathbb{D}$, Qing Kang ${ }^{2}$ and Kathryn E. Reif ${ }^{1, *(\mathbb{D})}$ \\ 1 Department of Diagnostic Medicine/Pathobiology, College of Veterinary Medicine, Kansas State University, \\ Manhattan, KS 66506, USA; ywikander@vet.k-state.edu \\ 2 Department of Statistics, College of Arts and Sciences, Kansas State University, Manhattan, KS 66506, USA; \\ qkang@k-state.edu \\ * Correspondence: kreif@vet.k-state.edu; Tel.: +1-216-201-0709
}

Received: 3 November 2020; Accepted: 15 December 2020; Published: 18 December 2020

\begin{abstract}
Cytauxzoon felis, a tick-borne hemoprotozoal pathogen of felids, causes an acute, often-fatal disease in domestic cats. While public awareness of the disease has increased, few studies have evaluated the incidence of acute cytauxzoonosis cases and their associated risk factors. The objective of this study was to retrospectively review records of cats diagnosed with acute cytauxzoonosis in eastern Kansas from 2006-2019 using clinic records and determine: (i) feline cytauxzoonosis risk factors; and (ii) if cytauxzoonosis case incidence is increasing. Although inter-annual variation of acute cytauxzoonosis diagnosis was observed in the eastern Kansas domestic cat population, the overall incidence trend remained largely unchanged over the 14-year case review period. In comparison to ill (C. felis-unrelated) control cases, more acute cytauxzoonosis cases were diagnosed in spring and summer, suggesting a seasonal fluctuation of infection, with samples most commonly submitted from $\geq 1$ year old, owned, male cats. Although cytauxzoonosis case submissions remained consistent over the broad study period, increasing tick vector and domestic cat reservoir populations may contribute to additional cytauxzoonosis case expansion in endemic areas. Investigating the incidence of acute cytauxzoonosis, patient risk factors, and ecological variables that influence disease transmission are important steps towards developing and communicating the need for effective cytauxzoonosis control strategies for high-risk cat populations.
\end{abstract}

Keywords: Cytauxzoon felis; cytauxzoonosis; domestic cat; fatal; piroplasm; schizont; signet ring

\section{Introduction}

Cytauxzoonosis is an often-fatal disease of domestic cats caused by Cytauxzoon felis, a tick-borne hemoprotozoal pathogen of felids. In the United States (U.S.), cytauxzoonosis cases most frequently occur in southeastern and south-central regions. This protozoal organism has a complex lifecycle that includes asexual reproduction in felid hosts and both asexual and sexual reproduction in competent ixodid tick vectors [1] (Figure 1). Briefly, C. felis sporozoites are transferred via tick saliva into a felid host during a blood meal. Once within the felid host, sporozoites enter monocytes and begin replicating asexually (schizogony) forming many 1-2 $\mu \mathrm{m}$ diameter signet ring merozoites. When the monocyte ruptures, these merozoites are released into the blood, where they enter host erythrocytes and begin either replicating asexually (merogony) or develop into gametocytes. Ticks become infected when they feed on an infected felid host and ingest gametocytes. The ingested gametocytes undergo sexual reproduction to form zygotes in the lumen of the tick gut. Zygotes invade the tick gut epithelium, transform into kinetes, and migrate to the salivary glands where they transform into sporozoites. 
Transstadial maintenance of the parasite through the larvae-to-nymph or nymph-to-adult ecdysis is required for the transmission of the infectious sporozoites to a new felid host during the subsequent tick bloodmeal. The competent biological transmission vectors of C. felis in the U.S. include Dermacentor variabilis (American dog tick) and Amblyomma americanum (Lone star tick) [2-5]. The range of D. variabilis encompasses the eastern U.S. as well as focal regions in the west [6,7]. The continuously expanding range of $A$. americanum covers the southeastern and mid-central U.S., largely overlapping with the range of $D$. variabilis [8,9]. Larval and nymphal $D$. variabilis prefer to feed on small to medium-sized mammals, whereas adults feed on larger mammals, including cats. In contrast, A. americanum are less discriminating, such that all life stages will seek out and feed on many mammals, including cats. Because of intensifying populations and its aggressive nature and willingness to feed on cats at all life stages, it is likely that $A$. americanum is a more significant vector of $C$. felis compared to $D$. variabilis in areas where both tick species reside.

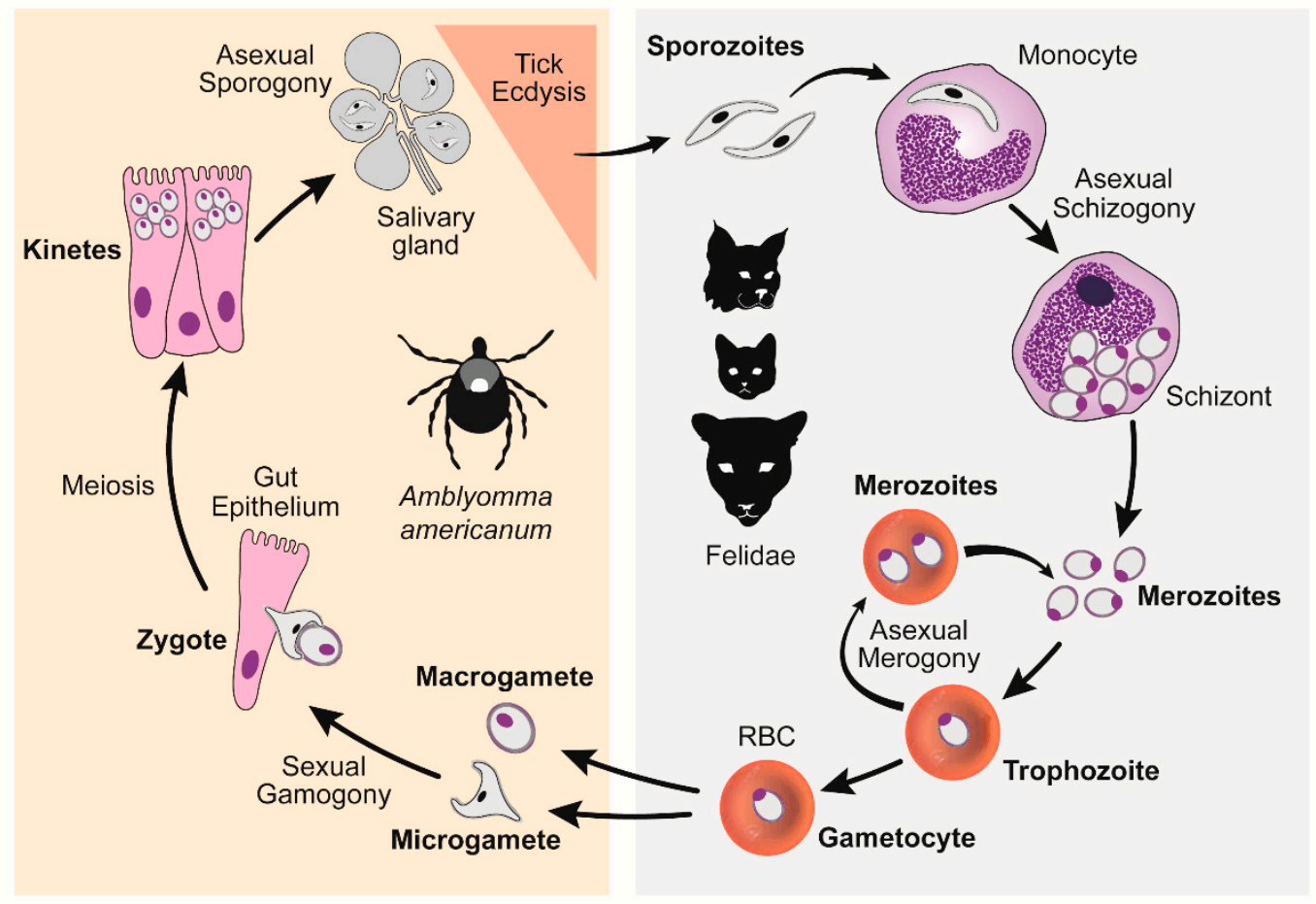

Figure 1. C. felis lifecycle. Right panel: C. felis reproduction within a felid host, asexual schizogony and merogony. Left panel: C. felis reproduction within a tick vector, sexual gamogony and asexual sporogony [10]. (Reproduced with permission from Yvonne Wikander, Pathogens; published by MDPI, 2020.).

Clinical cytauxzoonosis cases are most commonly observed in domestic cats (Felis catus), which serve as intermediate host carriers for $C$. felis along with other members of the Felidae family. Domestic cat cytauxzoonosis cases typically peak in late spring and early fall when the A. americanum adult and nymph life stages are most active [11]. For many decades, domestic cats were considered a dead-end host, as those with observed clinical disease commonly died [12-14]. Clinical disease signs for acute cytauxzoonosis appear during the schizogenous phase of $C$. felis asexual replication and can cause severe illness and death $[2,12]$. Cats commonly present with anorexia, depression, lethargy, pyrexia, dehydration, pale mucous membranes, and splenomegaly 11-13 days post infection (dpi) [2,15,16]. As the disease progresses, the pyrexia resolves and drops to subnormal, with icterus and dyspnea developing shortly thereafter. Complete blood count and serum biochemistry changes are seen around $13 \mathrm{dpi}$ and can include variable cytopenias, hypoalbuminemia, hyperglycemia, and increased ALT (alanine aminotransferase) and total bilirubin $[2,14,16]$. Intra-erythrocytic signet 
rings (also known as piroplasms) and/or schizont-laden monocytes are often observed at the feathered edge of thin blood smear preparations [2]. The organs most affected include the lung, liver, spleen, and lymph nodes $[13,14,16-18]$. Death occurs around 21 dpi due to inflammatory cytokine-mediated injury of multiple organs, interstitial pneumonia, hypoxic injury, and disseminated intravascular coagulation $[2,11,12,17-20]$. If initiated early in the disease process, treatment for ten days with a combination of atovaquone $(15 \mathrm{mg} / \mathrm{kg})$ and azithromycin $(10 \mathrm{mg} / \mathrm{kg})$ may result in the recovery of up to $60 \%$ of patients [21]. For survivors, clinical signs begin to resolve around 23-24 dpi, after which they become subclinical infected carriers that act as reservoirs for further $C$. felis transmission via competent tick vectors [2,22]. Domestic cat carriers of $C$. felis may be more common than previously thought, as we recently identified C. felis carrier infection in $25 \%$ of cats in eastern Kansas [10]. Similar clinical signs and disease progression have been reported with Cytauxzoon spp. in South America, Europe, and Asia [23-26].

Only a few studies have examined the incidence and risk factors associated with acute cytauxzoonosis cases in U.S. domestic cats; however, the conclusions of these studies vary. For example, investigation into cytauxzoonosis incidence trends varied from stable [11] to increasing [27], while evaluation of specific risk factors varied from identifying a predisposition of disease in young male cats [16] to tick climate and habitat conditions being more predictive of the disease rather than cat age and gender [28]. Anecdotal reports of increasing acute cytauxzoonosis cases, as well as heightened public awareness of this disease, makes understanding cytauxzoonosis and associated patient risk factors important for mitigating disease transmission among high-risk cat populations. To address these concerns in eastern Kansas, the objectives of this study were to: (i) determine if acute cytauxzoonosis cases in eastern Kansas increased between 2006-2019; and (ii) examine if specific feline risk factors are correlated with acute disease or disease outcomes, by performing a retrospective records search and review of acute cytauxzoonosis cases submitted to the Kansas State Veterinary Diagnostic Laboratory (KSVDL). Based on the high density of A. americanum ticks in eastern Kansas and feline risk factors reported as important by cytauxzoonosis studies in other states, we hypothesized that cases of acute cytauxzoonosis would: (i) show an increasing trend over time; (ii) peak in seasons of peak A. americanum activity; and (iii) be reported more commonly in young, intact, male cats. Updated information on acute cytauxzoonosis incidence and risk factors will help identify and focus C. felis education and intervention efforts for high-risk domestic cat populations.

\section{Materials and Methods}

\subsection{Ethic Approval}

All study activities involving animals and management of animal data were performed in accordance with an approved Kansas State University Institutional Animal Care and Use protocol (IACUC 4109), approved prior to study initiation.

\subsection{Study Design}

A retrospective study with individual cats as the study subject was designed using case records from the Kansas State Veterinary Diagnostic Laboratory (KSVDL) in Manhattan, Kansas. The KSVDL case database was queried for the term "Cytauxzoon felis" among records between May 2006 and October 2019 using the laboratory management software VetView v1.6.12 (University of Georgia, Athens, GA, USA). All search-populated records were individually reviewed to confirm that the cases met study inclusion criteria-(i) domestic cats from eastern Kansas, and (ii) a confirmed diagnosis of cytauxzoonosis. Cases that came from within the Kansas State University teaching hospital, as well as outside submissions of blood and/or tissue samples, were included in the study. Exclusion criteria included: (i) any cat not from eastern Kansas; or, (ii) cases where cytauxzoonosis diagnosis was not confirmed. Data collected from each feline patient record included: specimen receipt date (year, month, season); geographic information (county, state); diagnostic method (necropsy vs. blood smear); blood 
smear diagnosis (schizont vs. signet rings); signet ring frequency (occasional vs. frequent); patient age (years); patient sex (male vs. female) and sterilization status (neutral vs. intact); feline lifestyle (stray, owned, or rescue/rescinded); patient live/dead status within 30 days of initial case record; and mention of similar household cat deaths in the medical record (yes vs. no). Historical data of 123 control cases from Raghavan et al. [28], which consisted of "cats with a history of fever, malaise, icterus, and anorexia but no $C$. felis on blood film examination or schizonts within macrophages from fresh tissue or within multiple organs", were included as controls for this study [28].

\subsection{Statistical Analyses}

Yearly feline cytauxzoonosis incidence was analyzed under the Poisson log linear model with year block (2005 2009, 2010 1014, 2015 2019) being the fixed effect. Tukey's multiplicity adjustment was applied when comparing incidence rates among year blocks. For assessing risk factors including age ( $<1$ year, 1-3 years, 3-5 years, $\geq 5$ years) and season (Winter (December, January, Febuary), Spring (March, April, May), Summer (June, July, August), Fall (September, October, November)), the present study data of 170 cases were combined with the previously published 123 non-cytauxzoonosis control cases [28]. The resulting case-control data gave rise to two-way contingency tables where the Pearson chi-square test for difference in risk factor distributions was applied. For assessing sex (male, female) and lifestyle (feral, owned, rescue/rescinded) risk factors; diagnostic method used (blood smear, necropsy); C. felis life stages observed (signet rings, schizonts); and the relative amount of signet rings observed (frequent vs. occasional), one-way tables were created from the study data. A chi-square goodness-of-fit test was then performed to compare the observed percentages against the hypothesized distributions. When the cell count(s) in a contingency table were less than five, $p$ value was computed using the exact method instead of asymptotic approximation. The confidence interval for mortality rate was estimated using the Clopper-Pearson (exact) method. The confidence interval for percentage of owners of cats diagnosed with cytauxzoonosis reporting similar loss of other cats was estimated using the Wald method. Missing data were excluded from the statistical analyses. Statistical analyses were performed using the GENMOD and FREQ procedures of SAS/STAT ${ }^{\circledR}$ software, Version 9.4 (SAS Institute Inc., Cary, NC, USA).

\section{Results}

\subsection{Annual Acute Cytauxzoonosis Incidence in Domestic Cats from Eastern Kansas}

A total of 183 domestic cat records with a diagnosis of acute cytauxzoonosis from May 2006 through October 2019 were identified. Thirteen records were excluded from the study because they had no identifiable state of origin $(n=3)$ or they came from adjacent states $(n=10)$, leaving records from 170 domestic cats diagnosed with cytauxzoonosis from eastern Kansas available for analysis. These 170 records were evaluated to determine if the incidence of acute cytauxzoonosis cases was changing over time. Of those cases with a reported live-dead status $(n=140)$, only one cat survived. The greatest numbers of acute cytauxzoonosis cases were reported in 2006, 2009, 2012, 2017, 2018, and 2019 (Figure 2, Table S1). Because the number of cases varied from year-to-year, testing results were evaluated by year blocks: 2005-2009, 2010-2014, and 2015-2019. No significant difference was detected between any of the year blocks $(p=0.754)$ (Table 1, Table S2). Although acute cytauxzoonosis inter-annual variation was observed over time, collectively the incidence was stable over a broader time scale.

County-level locations of identified acute cytauxzoonosis cases in Kansas are presented in Figure 3. Acute cytauxzoonosis case samples were submitted to KSVDL from cats living in 31 eastern Kansas counties, with $>50 \%$ of the samples coming from the county in which the KSVDL resides (Riley) and those immediately adjacent (Pottawatomie, Wabaunsee, Geary, Clay, Marshall). Statistical analysis of location data for acute cytauxzoonosis cases in specific counties was not possible because the sampling method across counties was not standardized. 


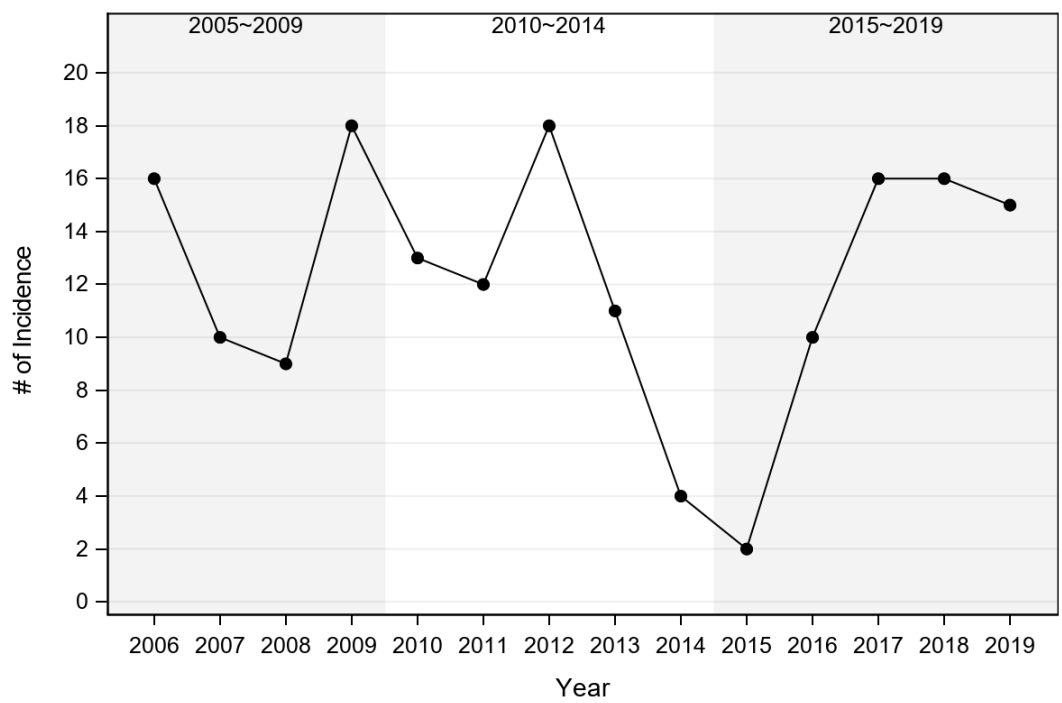

Figure 2. Incidence of acute cytauxzoonosis cases by year. Numbers represent raw acute cytauxzoonosis case counts.

Table 1. Statistical analysis of year block effect on acute cytauxzoonosis incidence.

\begin{tabular}{ccccc}
\hline & \multicolumn{2}{c}{ 1 of Incidence per Year } & \multicolumn{2}{c}{ Ratio to (Adj. $\boldsymbol{p}$ Value of Testing for Ratio $\neq \mathbf{1}$ ) } \\
\hline Year Block & Mean & SE $^{2}$ & 2010-2014 & 2015-2019 \\
\hline $2005 \sim 2009$ & 13.3 & 1.8 & $1.14(0.764)$ & $1.12(0.813)$ \\
$2010 \sim 2014$ & 11.6 & 1.5 & - & $0.98(0.995)$ \\
$2015 \sim 2019$ & 11.8 & 1.5 & - & - \\
\hline
\end{tabular}

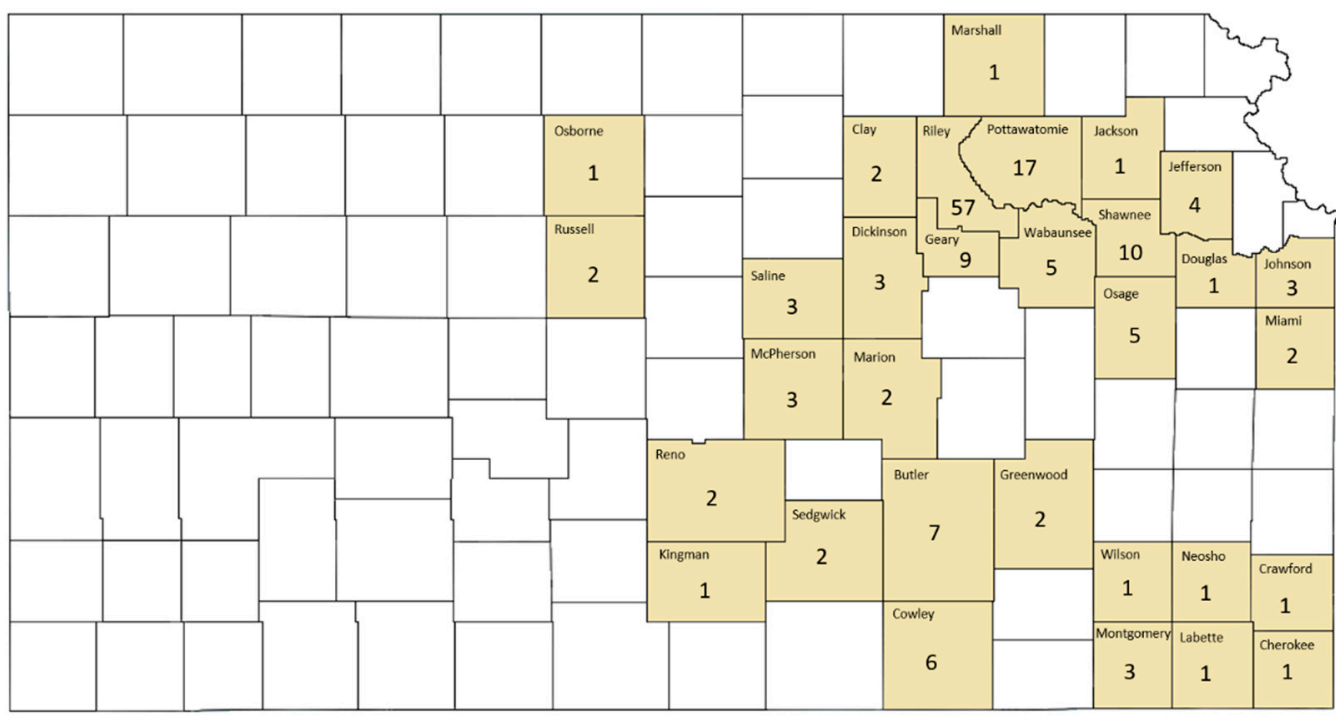

Figure 3. County-level location of acute cytauxzoonosis diagnosed domestic cat records in Kansas. Acute cytauxzoonosis case samples were submitted from cat(s) in shaded Kansas counties while no cat records were identified from unshaded counties. Numbers represent raw acute cytauxzoonosis case counts.

\subsection{Season Variation of Acute Cytauxzoonosis Incidence in Domestic Cats from Eastern Kansas}

To examine the intra-annual acute cytauxzoonosis case incidence, the incidence of acute cytauxzoonosis cases diagnosed in different months and seasons was evaluated (Figure 4). A bimodal 
distribution of cases was observed, with the greatest case peak between May $(n=40)$ and June $(n=51)$ and a second smaller case peak in September $(n=21)$. Similarly, the greatest proportion of acute cases was observed in summer (54.1\%), then spring (30.6\%) and fall (15.3\%). The seasonal distribution of acute cytauxzoonosis cases was significantly different from that of ill (C. felis-unrelated) control case incidence $(p<0.001)$ (Table 2, Figure S1). The seasons that contributed most to the significance (i.e., large Pearson chi-square statistic) are fall with $15.3 \%$ for the acute cytauxzoonosis cases vs. $38.2 \%$ for the ill control cases, as well as winter with $0 \%$ for the acute cytauxzoonosis cases vs. $7.3 \%$ for the ill control cases.

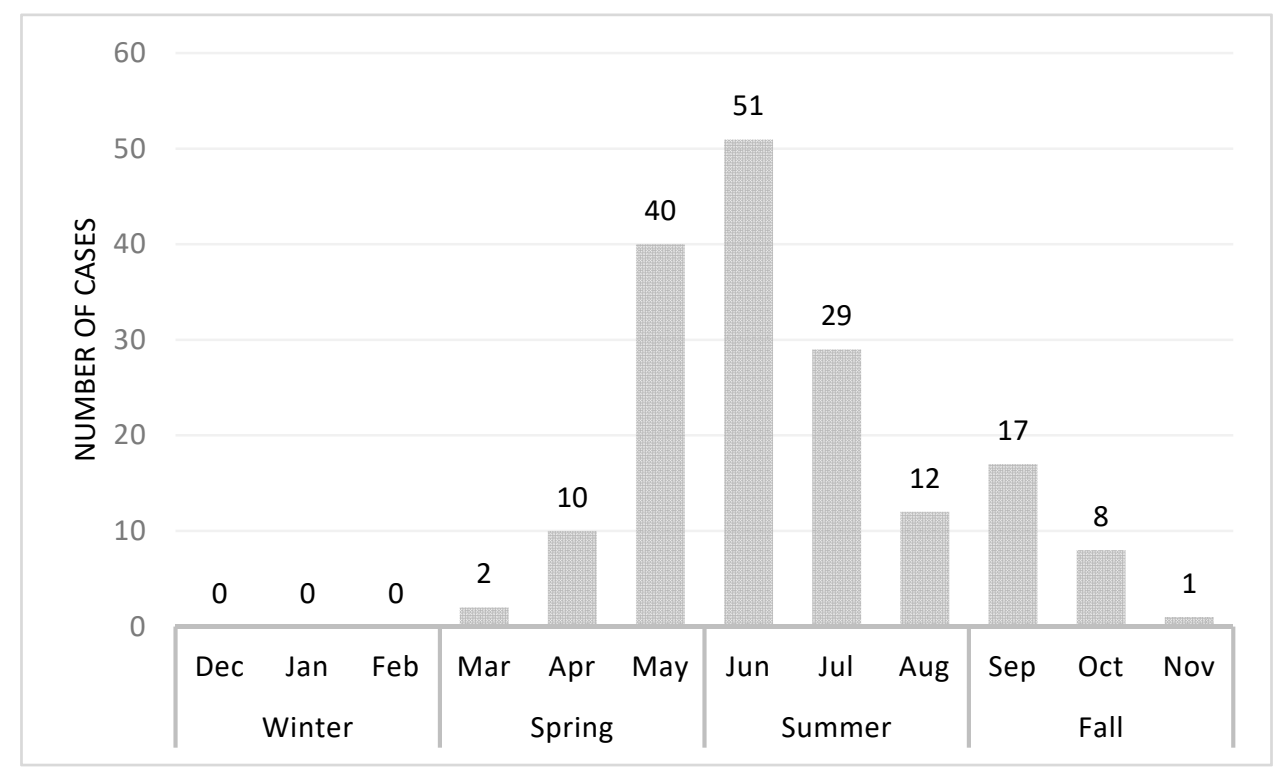

Figure 4. Acute cytauxzoonosis cases by month from May 2006 to Oct 2019. Case numbers represent the sum of total number of cases for each month from all years. These numbers represent raw acute cytauxzoonosis case counts.

Table 2. Statistical analysis of season effect on acute cytauxzoonosis and control case incidence.

\begin{tabular}{ccccccc}
\hline & \multicolumn{4}{c}{ Case } & \multicolumn{2}{c}{ Control } \\
\hline Effect & Season & Count & Percent & Count & Percent & $\boldsymbol{p}$ Value of Exact Chi-Square Test \\
\hline \multirow{2}{*}{ Season } & Spring & 52 & $30.6 \%$ & 21 & $17.1 \%$ & \\
& Summer & 92 & $54.1 \%$ & 46 & $37.4 \%$ & $<0.001$ \\
& Fall & 26 & $15.3 \%$ & 47 & $38.2 \%$ & \\
& Winter & 0 & $0.0 \%$ & 9 & $7.3 \%$ & \\
\hline
\end{tabular}

\subsection{Evaluation of Acute Cytauxzoonosis Incidence among Cats with Different Ages, Sex, and Lifestyle}

To examine if a correlation exists between specific cat risk factors with acute cytauxzoonosis case incidence, cat age, sex, and lifestyle were investigated. Feline age was reported in 156/170 cases evaluated, with the greatest number of acute cytauxzoonosis cases observed in cats 1-3 years old $(40.4 \%)$ and with fewer cases observed in $\geq 5$ years old $(25.6 \%), 3-5$ years old $(21.8 \%)$, and $<1$ year old $(12.2 \%)$ age groups (Table 3, Figure S2). The mean age of cats diagnosed with acute cytauxzoonosis was 3.4 years (range 1.4 months to 13 years) (Figure 5). The age distribution of acute cytauxzoonosis cases was significantly different from that of ill control case incidence $(p<0.001)$. The age group that contributed most to the significance (i.e., large Pearson chi-square statistic) is $<1$ year old with $12.2 \%$ for the acute cytauxzoonosis cases vs. $47.0 \%$ for the ill control cases. 
Table 3. Statistical analysis of age effect on acute cytauxzoonosis and control case incidence.

\begin{tabular}{ccccccc}
\hline & \multicolumn{6}{c}{ Case } \\
\hline \multirow{2}{*}{ Effect } & Age Group $^{\mathbf{1}}$ & Count $^{2}$ & Percent & Count & Percent & $\boldsymbol{p}$ Value of Chi-Square Test \\
\hline \multirow{4}{*}{ Age } & $<1$ & 19 & $12.2 \%$ & 47 & $47.0 \%$ & \\
& $1-3$ & 63 & $40.4 \%$ & 24 & $24.0 \%$ & $<0.001$ \\
& $3-5$ & 34 & $21.8 \%$ & 18 & $18.0 \%$ & \\
\hline
\end{tabular}

${ }^{1}$ Years, ${ }^{2}$ Age data were available and evaluated from 156/170 case records.

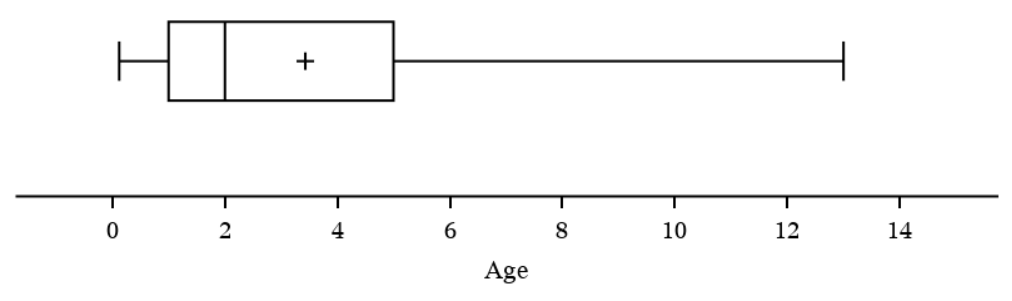

Figure 5. Acute cytauxzoonosis cases by age (years).

Feline sex was reported in 162/170 cases evaluated, with a diagnosis of acute cytauxzoonosis observed significantly more in male $(70.4 \%)$ compared to female $(29.6 \%)$ cats $(p<0.001)$ (Table 4$)$. The statistical analysis of sterilization status data was not possible as the sterilization status was not reported in the control group data [28] and data regarding sterilization status among the general Kansas cat population are unknown or unreported.

Table 4. Statistical analysis of sex effect on acute cytauxzoonosis case incidence.

\begin{tabular}{cccccc}
\hline \multicolumn{6}{c}{ Case } \\
\hline \multirow{2}{*}{ Effect } & Sex & Count $^{\mathbf{1}}$ & Percent & Percent Tested against & $\boldsymbol{p}$ Value of Chi-Square Test \\
\hline \multirow{2}{*}{ Sex } & F & 48 & $29.6 \%$ & $50.0 \%$ & $<0.001$ \\
& M & 114 & $70.4 \%$ & $50.0 \%$ & \\
\hline
\end{tabular}

${ }^{1}$ Sex data were available and evaluated from 156/170 case records.

Acute cytauxzoonosis cases were predominantly owned cats $(96.5 \%)$, with fewer feral and rescue/rescinded cats $(2.4 \%$ and $1.2 \%$, respectively) (Table 5$)$. The distribution of lifestyle for acute cytauxzoonosis was significantly different $(p<0.001)$ to that for the general cat population of Kansas, with $49.9 \%$ feral, $47.1 \%$ owned, and $3.0 \%$ rescue cats [29-32]. Collectively, acute cytauxzoonosis was most commonly observed in $\geq 1$ year old, male, owned cats.

Table 5. Statistical analysis of lifestyle effect on acute cytauxzoonosis case incidence.

\begin{tabular}{cccccc}
\hline \multicolumn{6}{c}{ Case } \\
\hline Effect & Lifestyle & Count & Percent & Percent Tested against & $p$ Value of Exact Chi-Square Test \\
\hline Lifestyle & feral & 4 & $2.4 \%$ & $3.0 \%$ & $<0.001$ \\
& owned & 164 & $96.5 \%$ & $47.1 \%$ & \\
& rescue & 2 & $1.2 \%$ & $49.9 \%$ & \\
\hline
\end{tabular}

\subsection{Evaluation of Method Used to Diagnose Acute Cytauxzoonosis in Domestic Cats from Eastern Kansas}

Lastly, the method used to diagnose acute cytauxzoonosis was evaluated (Table 6). Means of diagnosis did not vary significantly among acute cytauxzoonosis cases $(p=0.092)$; however, a small majority of cases were diagnosed via blood smear (56.5\%) vs. via necropsy results (43.5\%). Necropsy diagnosis was by visual identification of $C$. felis schizont(s) within vessels of any histological tissue 
sample. The tissues most commonly affected included the lung, liver, spleen, and lymph nodes. Blood smear diagnosis was made by visually identifying either the schizont (49\%) or intra-erythrocyte signet ring life stages (51\%) (also known as piroplasms) (Table 6). The blood smear diagnosis via schizont vs. signet rings did not vary significantly among acute cytauxzoonosis cases $(p=0.838)$. For those diagnosed through the identification of signet rings, the quantity of signet rings observed was generally classified as frequent $(61.2 \%)$ or occasional $(38.8 \%)$, which did not differ significantly from the 1:1 ratio $(p=0.116)$ (Table 6$)$. Based on cases with a known disease outcome (1 survivor and 139 deaths), the survival rate in acute cytauxzoonosis was $0.7 \%$ with $95 \%$ confidence interval of $(0.02 \%, 3.92 \%)$. The sole survivor was diagnosed by schizont identification using the blood smear method.

Table 6. Statistical analysis of diagnostic method, blood smear diagnosis, and relative number of signet ring form effects on acute cytauxzoonosis case mortality.

\begin{tabular}{|c|c|c|c|c|c|}
\hline \multicolumn{6}{|c|}{ Case } \\
\hline Effect & Group & Count & Percent & $\begin{array}{l}\text { Percent Tested } \\
\text { against }\end{array}$ & $\begin{array}{c}p \text { Value of } \\
\text { Chi-Square Test }\end{array}$ \\
\hline \multirow{2}{*}{ Method of Diagnosis } & blood smear & 96 & $56.5 \%$ & $50 \%$ & \multirow{2}{*}{0.092} \\
\hline & necropsy & 74 & $43.5 \%$ & $50 \%$ & \\
\hline \multirow{2}{*}{$\begin{array}{l}\text { Blood smear rings } \\
\text { vs. schizonts }\end{array}$} & rings & 49 & $51 \%$ & $50 \%$ & \multirow{2}{*}{0.838} \\
\hline & schizonts & 47 & $49 \%$ & $50 \%$ & \\
\hline \multirow{2}{*}{$\begin{array}{l}\text { Relative number of } \\
\text { rings observed }\end{array}$} & frequent & 30 & $61.2 \%$ & $50 \%$ & \multirow{2}{*}{0.116} \\
\hline & occasional & 19 & $38.8 \%$ & $50 \%$ & \\
\hline
\end{tabular}

\section{Discussion}

In C. felis endemic regions, acute cytauxzoonosis is one of the most fatal feline diseases. Several retrospective studies have demonstrated a bimodal pattern of seasonal cytauxzoonosis cases that correlates with $A$. americanum activity; young male cats being over-represented $[8,11,16,27,28,33]$. As a disease with a high fatality rate, increased public concern, and practitioner belief of increased incidence, understanding factors contributing to acute cytauxzoonosis can help focus efforts on preventing $C$. felis transmission among high-risk cat populations. In the present retrospective study, we demonstrate: (1) acute cytauxzoonosis case incidence in domestic cats from eastern Kansas has an overall stable trend; (2) acute cytauxzoonosis cases are more common in the spring and summer seasons; (3) young male cats are more likely to be diagnosed with acute cytauxzoonosis; and (4) schizont and/or intra-erythrocytic signet ring identification on histology or blood smear samples are reliable identifiers of acute cytauxzoonosis when associated with typical cytauxzoonosis clinical signs.

This study did not support anecdotal reports of an increasing acute cytauxzoonosis case trend in eastern Kansas cats based on KSVDL case sample submissions over the study period (Figure 2). Rather, the anecdotal reports of increasing acute cytauxzoonosis cases may be more attributed to individual year-to-year case fluctuations instead of a general increasing trend over the evaluated 14-year period (2006-2019). This finding agrees with a study that determined there was no significant year-to-year difference in 232 acute cytauxzoonosis cases identified over a 12-year period (1995-2006) in Oklahoma [11]. In contrast, another study determined there was an overall increase in acute cytauxzoonosis cases over a 10-year period (2001-2011) in western Kentucky [27]. However, the latter study consisted of only 56 cases and no statistical analysis method was reported. It is important to note that all three studies, including ours, were based on retrospectively evaluated data with limited patient details and histories. The discrepancy between anecdotal reports of increasing case numbers and data presented here may be due to a variety of reasons. Firstly, more clinicians may be diagnosing cytauxzoonosis with in-hospital microscopic blood smear evaluations versus sending out samples to a diagnostic laboratory, like KSVDL. Alternatively, an increased awareness of the disease may make the number of cases appear more prominent in the eyes of the clinicians, diagnosticians, and/or owners, while not actually being increased. Thirdly, vector tick populations-especially 
A. americanum - may be increasing or expanding their ranges with changing environmental conditions, increasing clinician index of suspicion and resulting in more suspected C. felis infection diagnoses without definitive diagnosis. Lastly, there may be an increasing incidence of acutely infected cats surviving infection without veterinary care, while the incidence of those presented to veterinary hospitals may be unchanged. Interestingly, with regard to this last point, we recently conducted a study investigating the prevalence of $C$. felis carrier (reservoir) cats in eastern Kansas and found that $25.8 \%$ of sampled domestic cats were actively infected with C. felis [10]. As such, more cats may survive acute cytauxzoonosis than previously expected, thus contributing to an expanding domestic cat reservoir population. The extent of $C$. felis genetic diversity and how these differences may affect virulence, transmissibility, and potential for treatment success is largely unexplored, with investigations hampered by a lack of methods to preserve or manipulate $C$. felis isolates outside of living cat or tick hosts. Attempts at identifying genetic markers of virulence have yet to find definitive virulence determinants [21,23,34-36].

A seasonal pattern of acute cytauxzoonosis incidence in domestic cats from eastern Kansas demonstrated a bimodal distribution, similar to those reported in other studies, peaking in early summer (June) with a smaller peak in early fall (September) $[11,16,27,28]$. This pattern corresponds with $A$. americanum peak activity in this area, which is predominantly dependent on environmental conditions like diurnal temperature range, precipitation, and humidity $[8,28]$. Adult ticks are most active in early spring to mid-summer, nymphs in late spring to mid-summer as well as late summer to early fall, and larvae in late summer to early fall [8]. This would suggest that $C$. felis may have been transmitted to the cats in this study predominantly by adults/nymphs in early summer with fewer transmissions occurring in early fall by nymphs. Previous tick transmission studies have demonstrated that both adult and nymphal $A$. americanum can successfully transmit $C$. felis $[2,11]$. However, since tick findings or tick preventative use practices were not identified in case records, further evaluation regarding the tick vector was not possible in this study. In addition, late spring through early fall may be times when cats spend more time outdoors actively roaming, resulting in a greater chance of encountering tick vectors. The combined seasonal cat and tick activity could explain why cats in our study area with evidence of fever, malaise, icterus, and anorexia were more likely to be diagnosed with acute cytauxzoonosis in spring/summer than other diseases with a similar presentation. A larger, long-range, prospective study evaluating both predisposing environmental conditions and habitat niches would provide insight into the timing of increased tick activity, which could be used to anticipate or forecast times of increased risk for $C$. felis transmission, as well as other tick-borne diseases.

As expected, young cats in their first and second year of life were more likely to have samples submitted and diagnosed with acute cytauxzoonosis. Our finding that young cats had a predisposition for acute cytauxzoonosis diagnosis supports previous data regarding age distribution for cats diagnosed with acute cytauxzoonosis [16] and tick infestation [33]. The first study, conducted in the mid-Atlantic states, found cats diagnosed with acute cytauxzoonosis $(n=34)$ had a mean age of 4 years (range 2 months to 14 years) [16]. Interestingly, another study investigating tick infestation of cats found cats with tick infestations were a mean age of 4.4 years (range 18 days to 18 years) $(n=336)$, with ticks recovered more frequently on young cats [33]. Combined, both studies support our study results of most commonly observing acute cytauxzoonosis cases in younger cats. In our study, a greater proportion of sample submissions came from young cats, for which there are several possible explanations. Firstly, young cats may explore their environment more aggressively and/or more frequently than older cats, resulting in potentially greater exposure to $C$. felis infected ticks. Secondly, owners may be more likely to present samples from younger cats with illness or an unexpected death, thinking an older cat died of old age. Thirdly, older cats may lack the energy or drive to return home when feeling ill and expire where their body is less likely to be retrieved. Lastly, older cats maybe be more likely to have encountered and survived a previous C. felis challenge, resulting in an asymptomatic reservoir stage. It is reasonable to assume that most cats that contract cytauxzoonosis do so through their outdoor activities because that is where the tick vector is most commonly located. 
Among cats diagnosed with acute cytauxzoonosis in our study, male cats were over-represented (Table 4), supporting a previous study that also found male cats to be over-represented (20/31; $64.5 \%$ male cats in other study) [16]. Interestingly, male cats were also previously found to be more likely to have tick infestations than female cats (59\% and $41 \%$, respectively) [33]. At one time it was believed that male cats had territories up to ten times larger than female cats, which could explain these findings [37]. However, more recent studies determined there is no significant difference in home range sizes between male and female cats [38-40]. As such, it is unclear why male cats had a higher incidence of disease compared to female cats in our study and others. A larger sample size as well as more complete patient background information would have been helpful in more thoroughly evaluating acute cytauxzoonosis risk factors; however, we were limited by the information provided in the case records. Overall, our findings regarding acute cytauxzoonosis seasonality and feline age/sex predilections in Kansas provide additional support to age and sex being important risk factors.

Not surprisingly, most of the sample submissions in this study came from owned cats $(96.5 \%)$, with rare rescue/rescinded or feral cat samples. Since owned cats generally benefit from a stronger human-animal bond, more diagnostics are performed either to understand the cause of a pet's demise or to ascertain if a zoonotic or infectious potential exists for the owner and/or other household pets. Additionally, 26.5\% (45/170) of the patient records evaluated included mention of multiple cats in the household dying of acute cytauxzoonosis or a cat that died in a similar but undiagnosed manner. These pet owners may be more sensitive to the potential for this disease in their pets, resulting in increased diagnostic efforts. Feral cats, barn cats, and/or outdoor "pets" which are fed but lack a strong human-animal bond with the owners are less likely to be submitted for diagnoses due to cost and/or difficulty in obtaining samples. This population of "wild" cats may also stray further afield, dying far from the owned property, or the property owners may be less attentive to these cats' cause of death or disappearance. Rescue and humane society organizations have limited medical care budgets and are more likely to perform on-site diagnostics for treatment decisions, and less likely to submit tissue samples to an outside diagnostic laboratory after death.

Rapid, patient-side diagnostics are not currently available for cytauxzoonosis diagnosis. Instead, the diagnosis of acute cytauxzoonosis is most commonly accomplished by a review of blood smears by a clinical pathologist or a review of tissue histology after necropsy. A diagnosis of acute cytauxzoonosis via identification of $C$. felis schizonts or intra-erythrocytic signet rings in blood smears, or schizonts in histology tissue samples, was equally diagnostic (Figure 6). Additionally, the density of intra-erythrocytic signet ring forms seen in blood smears did not determine the severity of this disease as nearly all cats in this cohort died or were euthanized (99.3\%) due to their illness. Different methods and criteria have been used in studies to diagnose acute cytauxzoonosis, including the identification of: (i) schizonts only [28]; (ii) schizonts or intra-erythrocytic signet rings [27]; or (iii) either schizonts or intra-erythrocytic signet rings with expected clinical signs [11]. Schizonts, when identified, are specific for acute cytauxzoonosis because this is the first life stage that develops in the feline host after the tick inoculates the sporozoites (Figure 1). In this study, observation of schizonts was noted in all case records where histologic tissue samples were evaluated (Figure 6C). That said, tissue sampling for histologic evaluation is far more costly and invasive than obtaining a blood sample, and requires a sedated, anesthetized, or deceased patient. Additionally, it does not demonstrate/confer a diagnostic advantage over a blood smear sample. Indeed, schizonts are not always identified on blood smear evaluation (Figure 6B). Approximately half of our blood smear samples lacked schizont identification. Piroplasms, $1-2 \mu \mathrm{m}$ diameter intra-erythrocyte signet rings, are found in both the schizogenous and erythrocytic phase of cytauxzoonosis and are too small to be reliably seen on histology (Figure 6A). Blood smear cases lacking schizonts in this study had either multiple intra-erythrocytic signet rings per erythrocyte (active merogony), a high density of intra-erythrocytic signet rings, or occasional intra-erythrocytic signet rings in patients exhibiting clinical signs consistent with acute cytauxzoonosis. These presenting cats were unlikely to be merely a $C$. felis reservoir, which can also have low parasite loads (generally $0.045-1.27 \%$ infected erythrocytes), because they also 
exhibited clinical signs of cytauxzoonosis [22]. In subacute and early acute infections, intra-erythrocytic signet rings and schizonts may not be immediately apparent on blood smears. Blood or tissue sample submissions to a diagnostic laboratory for pathologist evaluation takes time that these patients may not have. Ideally, a rapid chairside test for in-house veterinary use would be developed to identify these cases very early in the disease process, allowing for early treatment initiation that may prove lifesaving for many of these cats.

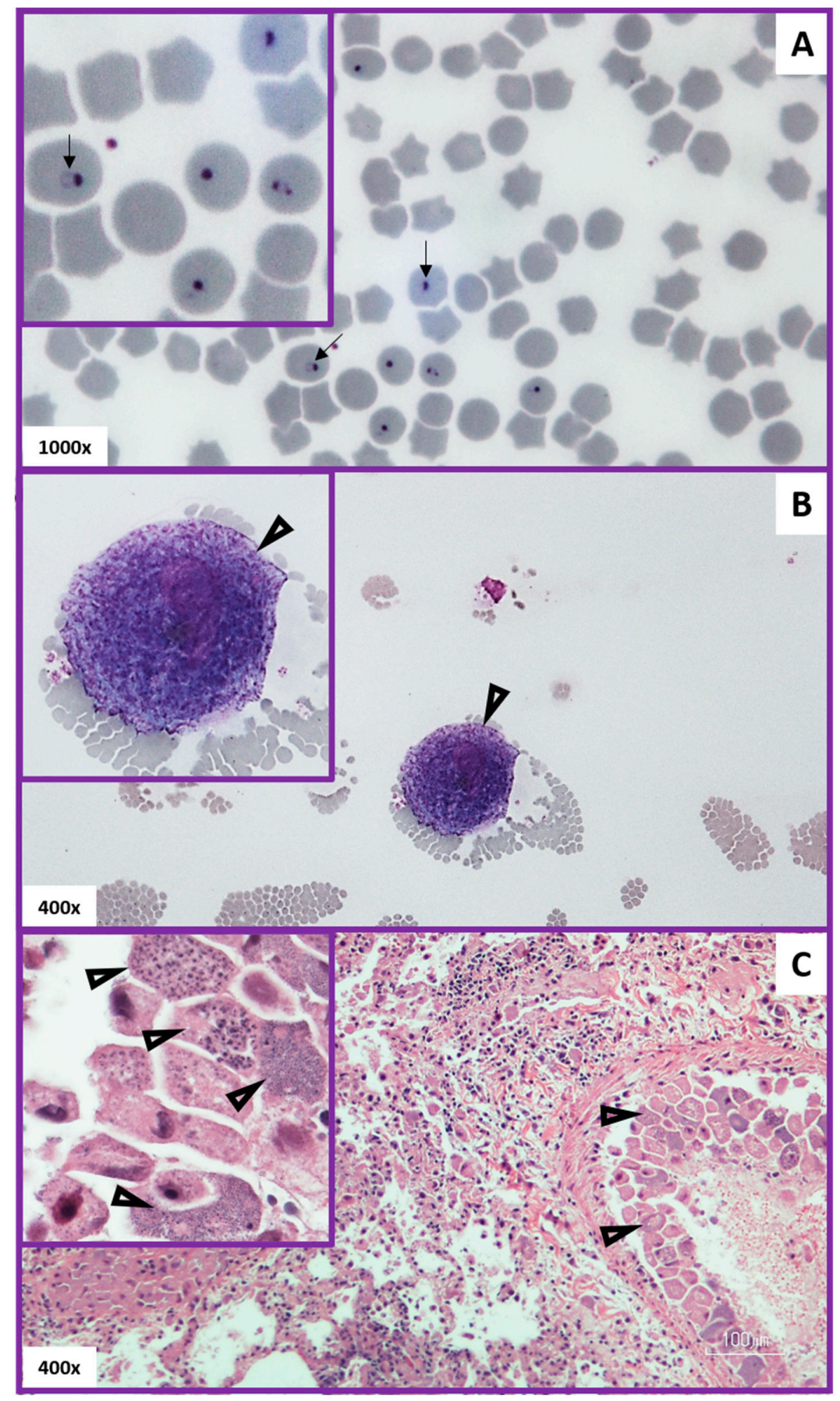

Figure 6. Diagnostic pathology of acute cytauxzoonosis. Panel (A) (top): Blood smear with intra-erythrocytic signet rings (arrows). Panel (B) (middle): Blood smear with a schizogenous monocyte (schizont) at the feathered edge (open arrowheads). Panel (C) (bottom): Histologic lung tissue with schizonts attached to endothelium (open arrowheads) within a vessel. Note the variable schizont developmental stages (arrows) seen in the Panel C inset. 
The differential diagnosis for the nonspecific clinical signs seen in cytauxzoonosis (lethargy, fever, icterus, dyspnea, +/- anemia) could include cholangiohepatitis, triaditis, pancreatitis, hepatic lipidosis, sepsis, immune-mediated hemolytic anemia, toxins causing oxidative damage (e.g., Allium spp" acetaminophen), neoplasia, and infectious agents (tularemia, feline infectious peritonitis, hemotropic mycoplasma), to name a few [41,42]. When intra-erythrocytic hemoparasites with a signet ring or piroplasm appearance are seen in cats, there are three main differential diagnoses: (i) Cytauxzoon felis, (ii) Mycoplasma hemofelis, and (iii) Babesia felis. C. felis, discussed herein, typically presents with a variable non-regenerative anemia, intra-erythrocytic signet rings, rare to occasional signet rings in the background, and/or schizonts at the feathered edge of blood smears or on histology of tissue samples. M. hemofelis commonly presents with a strong regenerative anemia, and signet ring and/or coccoid forms of epierythrocytic organism located on the red cells and/or in the background [41]. B. felis presents with a regenerative anemia and intra-erythrocytic signet rings often arranged in tetrads [42]. Considering our study area is within the U.S., B. felis is unlikely to be the cause of infection in these cats as B. felis has not been reported in the U.S. However, it should be considered for any feline patient with a travel history to Africa, particularly the southern coastal regions [42]. While M. hemofelis and Babesia spp. PCR is readily available for differentiation, C. felis PCR is less available. That said, identification of either schizonts and/or intra-erythrocytic signet rings via histology or blood smear samples are equally diagnostic for acute cytauxzoonosis when associated with typical acute cytauxzoonosis clinical signs for cats living in or near endemic areas. In locations where these feline piroplasm diseases overlap, DNA sequencing may be necessary to resolve piroplasm species identity.

\section{Conclusions}

In conclusion, we determined the incidence of acute cytauxzoonosis in domestic cats of eastern Kansas has remained stable over the last 14 years, commonly occurs in young male cats, and correlates with the expected A. americanum activity and life cycle. Clinicians practicing in or near endemic areas should consider acute cytauxzoonosis as a differential for any cat exhibiting depression, lethargy, fever, anorexia, icterus, anemia, cytopenias, or sudden death with confirmation via histologic or blood smear identification of schizonts or intra-erythrocytic signet rings and/or PCR. Since no vaccine exists and effective treatment options are limited, $C$. felis tick transmission should be mitigated by the use of aggressive, year-around, acaricide products for all domestic cats living in endemic areas, regardless of age, sex, or lifestyle. More studies are needed to further elucidate factors affecting cytauxzoonosis disease progression and presentation, and treatment options and outcomes within the U.S. and globally.

Supplementary Materials: The following are available online at http://www.mdpi.com/2306-7381/7/4/205/s1, Table S1: Acute cytauxzoonosis case incidence by year and year block, Table S2: Acute cytauxzoonosis case incidence by year block, Figure S1: Acute cytauxzoonosis and control case percentages by season, Figure S2: Acute cytauxzoonosis and control case percentage by age group (years).

Author Contributions: Conceptualization, K.E.R.; methodology, K.E.R.; software, Q.K.; validation, Y.M.W. and K.E.R.; formal analysis, Q.K.; investigation, Y.M.W.; resources, K.E.R.; data curation, Y.M.W.; writing-original draft preparation, Y.M.W., K.E.R. and Q.K.; writing-review and editing, Y.M.W., K.E.R., and Q.K.; visualization, Y.M.W.; supervision, K.E.R.; project administration, Y.M.W. and K.E.R.; funding acquisition, K.E.R. All authors have read and agreed to the published version of the manuscript.

Funding: This research received no external funding.

Acknowledgments: We would like to thank Mal Rooks Hoover, Certified Medical Illustrator, for her assistance in figure preparation.

Conflicts of Interest: The authors declare no conflict of interest. 


\section{References}

1. Jalovecká, M.; Hajdusek, O.; Sojka, D.; Kopacek, P.; Malandrin, L. The complexity of piroplasms life cycles. Front. Cell. Infect. Microbiol. 2018, 8, 248. [CrossRef] [PubMed]

2. Reichard, M.V.; Meinkoth, J.H.; Edwards, A.C.; Snider, T.A.; Kocan, K.M.; Blouin, E.F.; Little, S.E. Transmission of Cytauxzoon felis to a domestic cat by Amblyomma americanum. Vet. Parasitol. 2009, 161, 110-115. [CrossRef] [PubMed]

3. Thomas, J.E.; Ohmes, C.M.; Payton, M.E.; Hostetler, J.A.; Reichard, M.V. Minimum transmission time of Cytauxzoon felis by Amblyomma americanum to domestic cats in relation to duration of infestation, and investigation of ingestion of infected ticks as a potential route of transmission. J. Feline Med. Surg. 2018, 20, 67-72. [CrossRef] [PubMed]

4. Allen, K.E.; Thomas, J.E.; Wohltjen, M.L.; Reichard, M.V. Transmission of Cytauxzoon felis to domestic cats by Amblyomma americanum nymphs. Parasites Vectors 2019, 12, 28. [CrossRef] [PubMed]

5. Little, S.E.; Barrett, A.W.; Nagamori, Y.; Herrin, B.H.; Normile, D.; Heaney, K.; Armstrong, R. Ticks from cats in the United States: Patterns of infestation and infection with pathogens. Vet. Parasitol. 2018, 257, 15-20. [CrossRef] [PubMed]

6. Chan, W.-H.; Kaufman, P.E. Common Name: American Dog Tick Scientific Name: Dermacentor variabilis (Say) (Arachnida: Ixodida: Ixodidae). Available online: http://entnemdept.ufl.edu/creatures/urban/medical/ american_dog_tick.htm (accessed on 24 March 2020).

7. Minigan, J.N.; Hager, H.A.; Peregrine, A.S.; Newman, J.A. Current and potential future distribution of the American dog tick (Dermacentor variabilis, Say) in North America. Ticks Tick-Borne Dis. 2018, 9, 354-362. [CrossRef]

8. Holderman, C.J.; Kaufman, P.E. Common Name: Lone Star Tick Scientific Name: Amblyomma americanum (Linnaeus) (Acari: Ixodidae). Available online: http://entnemdept.ufl.edu/creatures/urban/medical/lone_ star_tick.htm (accessed on 15 March 2020).

9. Monzón, J.D.; Atkinson, E.G.; Henn, B.M.; Benach, J.L. Population and evolutionary genomics of Amblyomma americanum, an expanding arthropod disease vector. Genome Biol. Evol. 2016, 8, 1351-1360. [CrossRef]

10. Wikander, Y.M.; Anantatat, T.; Kang, Q.; Reif, K.E. Prevalence of Cytauxzoon felis infection-carriers in eastern Kansas domestic cats. Pathogens 2020, 9, 854. [CrossRef]

11. Reichard, M.V.; Baum, K.A.; Cadenhead, S.C.; Snider, T.A. Temporal occurrence and environmental risk factors associated with cytauxzoonosis in domestic cats. Vet. Parasitol. 2008, 152, 314-320. [CrossRef]

12. Ferris, D. A progress report on the status of a new disease of American cats: Cytauxzoonosis. Comp. Immunol. Microbiol. Infect. Dis. 1979, 1, 269-276. [CrossRef]

13. Wagner, J.E. A fatal cytauxzoonosis-like disease in cats. J. Am. Vet. Med Assoc. 1976, 168, 585-588. [PubMed]

14. Hoover, J.P.; Walker, D.B.; Hedges, J.D. Cytauxzoonosis in cats: Eight cases (1985-1992). J. Am. Vet. Med. Assoc. 1994, 205, 455-460. [PubMed]

15. Meinkoth, J.; Kocan, A.A.; Whitworth, L.; Murphy, G.; Fox, J.C.; Woods, J.P. Cats surviving natural infection with Cytauxzoon felis: 18 cases (1997-1998). J. Vet. Intern. Med. 2000, 14, 521-525. [CrossRef]

16. Birkenheuer, A.J.; Le, J.A.; Valenzisi, A.M.; Tucker, M.D.; Levy, M.G.; Breitschwerdt, E.B. Cytauxzoon felis infection in cats in the mid-Atlantic states: 34 cases (1998-2004). J. Am. Vet. Med. Assoc. 2006, 228, 568-571. [CrossRef] [PubMed]

17. Jackson, C.B.; Fisher, T. Fatal cytauxzoonosis in a Kentucky cat (Felis domesticus). Vet. Parasitol. 2006, 139, 192-195. [CrossRef] [PubMed]

18. Snider, T.A.; Confer, A.W.; Payton, M.E. Pulmonary Histopathology of Cytauxzoon felis infections in the cat. Vet. Pathol. 2010, 47, 698-702. [CrossRef] [PubMed]

19. Frontera-Acevedo, K. Feline Immune Response to Infection with Cytauxzoon felis and the Role of CD18 in the Pathogenesis of Cytauxzoonosis. Ph.D. Thesis, University of Georgia, Athens, GA, USA, 2013.

20. Conner, B.J.; Hanel, R.M.; Brooks, M.B.; Cohn, L.A.; Birkenheuer, A.J. Coagulation abnormalities in 5 cats with naturally occurring cytauxzoonosis. J. Vet. Emerg. Crit. Care 2015, 25, 538-545. [CrossRef]

21. Cohn, L.A.; Birkenheuer, A.; Brunker, J.; Ratcliff, E.; Craig, A. Efficacy of atovaquone and azithromycin or imidocarb dipropionate in cats with acute cytauxzoonosis. J. Vet. Intern. Med. 2010, 25, 55-60. [CrossRef]

22. Wang, J.-L.; Li, T.-T.; Liu, G.-H.; Zhu, X.-Q.; Yao, C.-Q. Two tales of Cytauxzoon felis infections in domestic cats. Clin. Microbiol. Rev. 2017, 30, 861-885. [CrossRef]

23. Zou, F.-C.; Li, Z.; Yang, J.-F.; Chang, J.-Y.; Liu, G.-H.; Lv, Y.; Zhu, X.-Q. Cytauxzoon felis infection in domestic cats, Yunnan Province, China, 2016. Emerg. Infect. Dis. 2019, 25, 353-354. [CrossRef] 
24. Furtado, M.M.; Taniwaki, S.A.; Metzger, B.; Paduan, K.D.S.; O’Dwyer, H.L.; Jácomo, A.T.D.A.; Porfírio, G.E.; Silveira, L.; Sollmann, R.; Tôrres, N.M.; et al. Is the free-ranging jaguar (Panthera onca) a reservoir for Cytauxzoon felis in Brazil? Ticks Tick-Borne Dis. 2017, 8, 470-476. [CrossRef] [PubMed]

25. Legroux, J.-P.; Halos, L.; René-Martellet, M.; Servonnet, M.; Pingret, J.-L.; Bourdoiseau, G.; Baneth, G.; Chabanne, L. First clinical case report of Cytauxzoon sp. infection in a domestic cat in France. BMC Vet. Res. 2017, 13, 81. [CrossRef]

26. Veronesi, F.; Ravagnan, S.; Cerquetella, M.; Carli, E.; Olivieri, E.; Santoro, A.; Pesaro, S.; Berardi, S.; Rossi, G.; Ragni, B.; et al. First detection of Cytauxzoon spp. infection in European wildcats (Felis silvestris silvestris) of Italy. Ticks Tick-Borne Dis. 2016, 7, 853-858. [CrossRef] [PubMed]

27. Miller, J.; Davis, C. Increasing frequency of feline cytauxzoonosis cases diagnosed in western Kentucky from 2001 to 2011. Vet. Parasitol. 2013, 198, 205-208. [CrossRef] [PubMed]

28. Raghavan, R.K.; Almes, K.M.; Goodin, D.G.; Harrington, J.J.A.; Stackhouse, J.P.W. Spatially heterogeneous land cover/land use and climatic risk factors of tick-borne feline cytauxzoonosis. Vector-Borne Zoonotic Dis. 2014, 14, 486-495. [CrossRef] [PubMed]

29. Pets by the Numbers: U.S. Pet Ownership, Community Cat and Shelter Population Sstimates. Available online: https://www.humanesociety.org/resources/pets-numbers (accessed on 10 August 2020).

30. Pets by the Numbers: Data and Statistics on Pet Ownership, Community Cat, and Shelter Population in the United States. Available online: https://www.animalsheltering.org/page/pets-by-the-numbers (accessed on 10 August 2020).

31. Gedeon, J. Special Report: States with the Most and Least Cat Owners. Available online: https://247wallst. com/special-report/2017/07/19/states-with-the-most-and-least-cat-owners/ (accessed on 10 August 2020).

32. History of National Feral Cat Day. Available online: https://nationaltoday.com/national-feral-cat-day/ (accessed on 10 August 2020).

33. Saleh, M.N.; Sundstrom, K.D.; Duncan, K.T.; Ientile, M.M.; Jordy, J.; Ghosh, P.; Little, S.E. Show us your ticks: A survey of ticks infesting dogs and cats across the USA. Parasites Vectors 2019, 12, 1-11. [CrossRef] [PubMed]

34. Schreeg, M.E.; Marr, H.S.; Tarigo, J.; Cohn, L.A.; Levy, M.G.; Birkenheuer, A.J. Pharmacogenomics of Cytauxzoon felis cytochrome b: Implications for atovaquone and azithromycin therapy in domestic cats with cytauxzoonosis. J. Clin. Microbiol. 2013, 51, 3066-3069. [CrossRef] [PubMed]

35. Brown, H.M.; Modaresi, S.M.; Cook, J.L.; Latimer, K.S.; Peterson, D.S. Genetic variability of archived Cytauxzoon felis histologic specimens from domestic cats in Georgia, 1995-2007. J. Vet. Diagn. Investig. 2009, 21, 493-498. [CrossRef]

36. Shock, B.C.; Birkenheuer, A.J.; Patton, L.L.; Olfenbuttel, C.; Beringer, J.; Grove, D.M.; Peek, M.; Butfiloski, J.W.; Hughes, D.W.; Lockhart, J.M.; et al. Variation in the ITS-1 and ITS-2 rRNA genomic regions of Cytauxzoon felis from bobcats and pumas in the eastern United States and comparison with sequences from domestic cats. Vet. Parasitol. 2012, 190, 29-35. [CrossRef]

37. The Territory of Outdoor Cats. Available online: https://www.knowyourcat.info/info/teritory.htm (accessed on 31 August 2020).

38. Meek, P. Home range of house cats Felis catus living within a National Park. Aust. Mammal. 2003, 25, 51-60. [CrossRef]

39. Horn, J.A.; Mateus-Pinilla, N.; Warner, R.E.; Heske, E.J. Home range, habitat use, and activity patterns of free-roaming domestic cats. J. Wildl. Manag. 2011, 75, 1177-1185. [CrossRef]

40. Hanmer, H.J.; Thomas, R.L.; Fellowes, M.D.E. Urbanization influences range size of the domestic cat (Felis catus): Consequences for conservation. J. Urban Ecol. 2017, 3, 1-11. [CrossRef]

41. Sykes, J. Feline hemotropic mycoplasmas. Vet. Clin. North Am. Small Anim. Pr. 2010, 40, 1157-1170. [CrossRef] [PubMed]

42. Penzhorn, B.L.; Oosthuizen, M.C. Babesia species of domestic cats: Molecular characterization has opened Pandora's box. Front. Vet. Sci. 2020, 7, 1-10. [CrossRef] [PubMed]

Publisher's Note: MDPI stays neutral with regard to jurisdictional claims in published maps and institutional affiliations. 